Classification

Physics Abstracts

$68.10 \mathrm{C}-68.45-47.55 \mathrm{~K}-47.55 \mathrm{M}$

\title{
Effets de la géométrie des pores et de la mouillabilité sur le déplacement diphasique à contre-courant en capillaire et en milieu poreux
}

\author{
François Kalaydjian et Benoît Legait \\ Institut Français du Pétrole, B.P. 311, 92506 Rueil-Malmaison Cedex, France \\ (Reçu le 10 septembre 1987, révisé le 16 décembre 1987, accepté le 3 mars 1988)
}

\begin{abstract}
Résumé. - Le déplacement à contre-courant en imbibition spontanée d'un globule de fluide non mouillant est étudié dans un capillaire de section carrée. La modélisation montre les effets de la géométrie du système et de l'angle de mouillage sur la dynamique du processus et sur l'origine d'un couplage entre phases fluides qui nécessite une nouvelle formulation des lois d'écoulement en milieu poreux.

Abstract. - Counter-current flow of a non-wetting ganglion during spontaneous imbibition, is studied in a capillary tube of square cross-section. Laboratory experiments and calculations underline the effects of geometry and contact angle on the flow dynamics and lead to a new formulation of laws of diphasic diplacement in porous media, taking into account coupling between fluid phases.
\end{abstract}

\section{Notations.}

\section{Lettres latines}

$\left(B_{i}^{j}\right)$ matrice inverse de $\left(C_{i}^{j}\right)$ (cf. Eq. (3))

$C(z)$ courbure de l'interface fluide/fluide

$C_{i}$ courbure du ménisque $\Sigma_{i} ; i=1,2$

$\left(C_{i}^{j}\right)$ matrice de flux (cf. annexe 1)

$k$ perméabilité intrinsèque du milieu

$k_{i} \quad$ mobilité de la phase $i=1,2$

$k_{r i}$ perméabilité relative de la phase $i=1,2$

$k_{r i j}$ coefficient de la matrice des perméabilités $i, j=1,2$

$k_{i j}$ coefficient de la matrice des mobilités $i, j=1,2$

$\mathcal{L} \quad$ interface fluide/fluide dans une section transverse

$N_{\text {c }} \quad$ nombre capillaire

$P_{i} \quad$ pression exercée par le système sur la phase $i=1,2$

$P_{\mathrm{c}} \quad$ pression capillaire $\left(P_{\mathrm{c}}=P_{1}-P_{2}\right)$

$q_{i} \quad$ débit de la phase $i=1,2$

$Q \quad$ valeur absolue de $q_{i}$

$r(z)$ demi-côté d'une section du capillaire à la cote $z$ $s_{i} \quad$ saturation de la phase $i=1,2$

$S_{i} \quad$ section du capillaire à la position $Z_{i}, i=1,2$

$S \quad$ surface du solide

$T$ température du système

$\mathbf{u}_{i} \quad$ vitesse de la phase $i=1,2$

$Z_{i}$ position du ménisque $\Sigma_{i}, i=1,2$

\section{Lettres grecques}

$\alpha \quad$ fonction de $\bar{\mu}$ et $\theta$ (cf. Eq. (10))

$\beta \quad$ facteur de forme (cf. annexe 2)

$\gamma$ tension interfaciale de l'interface fluide/fluide

$\lambda_{i} \quad$ constante de flux (cf. Eq. (7)) $i=1,2$

$\mu_{i} \quad$ viscosité de la phase fluide $i=1,2$

$\bar{\mu} \quad$ contraste de viscosité : $\bar{\mu}=\mu_{1} / \mu_{2}$

$\rho_{i}$ masse volumique de la phase fluide $i=1,2$

$\theta$ angle de mouillage

$\Sigma_{i} \quad$ ménisque $i$ du globule d'huile $i=1,2$

\section{Indices}

1 fluide non mouillant

2 fluide mouillant 


\section{Introduction.}

De nombreux exemples d'écoulements polyphasiques en milieu poreux proviennent des problèmes liés à la récupération du pétrole. Une description fine des processus physico-chimiques mis en jeu, est nécessaire pour optimiser le taux de récupération. Ceci a motivé de multiples études tant à l'échelle microscopique, celle des pores, qu'à l'échelle macroscopique, celle du milieu poreux : la compréhension et la prédiction à l'échelle macroscopique des mécanismes qui contrôlent le déplacement des fluides passe souvent par des études à l'échelle microscopique pour laquelle le formalisme est plus rigoureux.

En effet, si à l'échelle microscopique, l'équation de Navier-Stokes régit les écoulements, leur description à l'échelle macroscopique est moins rigoureuse : à cette échelle, l'écoulement est décrit par la loi de Darcy qui a été établie, dans le cas monophasique de façon empirique (Darcy, 1856). Cette loi gouverne l'écoulement d'un fluide à travers un milieu poreux sous l'effet des forces de gravité et de viscosité, la perméabilité intrinsèque du milieu étant à un facteur multiplicatif près le rapport de proportionnalité entre la charge hydraulique imposée et le débit résultant.

Cette loi peut dans le cas monophasique être retrouvée de façon théorique, moyennant quelques simplifications, à l'aide par exemple de la thermodynamique des processus irréversibles (Marle, 1967).

La généralisation de cette loi au cas polyphasique (Wyckoff, 1936), appelée loi de Darcy généralisée, induit de même la notion de perméabilité relative à chacun des fluides, supposée ne dépendre explicitement que de l'état de saturation du milieu, notion dont la justification théorique n'est pas immédiate. Ainsi, Rose (1971) souligne que le fait d'utiliser la loi de Darcy dans le cas polyphasique revient à considérer qu'un milieu poreux dans lequel circulent deux fluides immiscibles est censé constituer un nouveau milieu poreux pour le second. Cette hypothèse conduit à écrire une même condition limite aux interfaces fluide/fluide et fluide/solide.

De plus, Lelièvre (1966) a montré expérimentalement que les courbes de perméabilités relatives dépendent du type d'écoulement considéré, ce dont ne rend pas compte la modélisation à partir de la loi de Darcy. Ainsi, selon que les fluides se déplacent à co-courant ou à contre-courant, les perméabilités relatives ne sont pas identiques, celles à contrecourant étant notablement inférieures. Ceci provient en fait de ce que les mécanismes physiques de déplacement sont fondamentalement différents dans les deux cas. Dans le premier cas, celui du cocourant, le déplacement est essentiellement de type piston, un fluide poussant l'autre, tandis que dans le second cas, le mécanisme est plus complexe et fait intervenir outre l'effet piston qui induit un contrecourant macroscopique (les deux fluides cheminant alors par des circuits différents), un effet capillaire de couplage entre les deux fluides dû à la géométrie anguleuse des pores et à la mouillabilité différente de fluides vis-à-vis du milieu poreux: le fluide mouillant progresse par capillarité le long des parois des pores et mobilise la phase non mouillante qui peut ainsi cheminer par la partie centrale des mêmes pores (Lenormand, 1981 ; Lenormand et al., 1984 ; Kalaydjian et al., 1987a).

Un tel déplacement diphasique à contre-courant peut avoir lieu lors de la récupération d'huile dans des gisements fissurés, par circulation d'eau dans les fissures. Au voisinage de celles-ci, et pour un milieu mouillable à l'eau, le phénomène d'imbibition spontanée, i.e. l'envahissement du milieu poreux par l'eau sous l'action de la capillarité entraîne une production d'huile par contre-courant.

Une étude plus fine du contre-courant, à l'échelle du pore, permettant d'isoler cette notion de couplage et de clarifier ainsi l'usage de la notion de perméabilité relative s'avère donc nécessaire.

Tel est le but de cette étude, restreinte au cas d'écoulements diphasiques incompressibles au sein de milieux poreux mouillables à l'eau et qui s'organise comme suit. Dans une première partie, nous décrivons le micromodèle que nous avons utilisé et nous présentons la procédure expérimentale mise en œuvre. Dans une seconde partie, nous présentons les résultats expérimentaux qui, confrontés à la théorie dans une troisième partie, permettent de dégager clairement l'influence de certains paramètres physico-chimiques tels que la mouillabilité, la tension interfaciale, le rapport des viscosités des fluides, sur la dynamique du processus. Nous montrons dans une quatrième partie comment l'interprétation des résultats expérimentaux obtenus sur capillaires, en termes de perméabilité relative permet d'exhiber la notion de couplage de perméabilité qui est ensuite quantifiée. Cette notion est enfin appliquée au cas d'un milieu poreux.

\section{Approche expérimentale.}

Toutes les expériences ont été effectuées à une température maintenue à $T=30 \pm 0.5^{\circ} \mathrm{C}$.

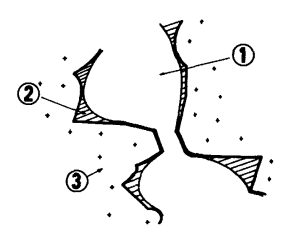

Fig. 1. - Répartition des fluides à l'échelle du pore : 1 fluide non mouillant ; 2 fluide mouillant ; 3 phase solide.

[Fluid distribution at pore scale : 1 non-wetting fluid ; 2 wetting fluid ; 3 solid phase.] 
1.1 Choix DU CAPILlaire. - A l'échelle du pore, du fait de l'angulosité des pores, l'huile et l'eau se répartissent de telle façon que l'eau occupe les anfractuosités des parois des pores, tandis que l'huile occupe le centre des pores (Fig. 1). Le capillaire servant de support à l'étude microscopique d'un écoulement diphasique à contre-courant doit donc avoir une section anguleuse. Singhal et al. (1970) ont ainsi utilisé des capillaires de section triangulaire. Mais un calcul simple montrant que pour un même angle de mouillage, le domaine occupé par le fluide mouillant est plus important dans le cas d'une section carrée que dans celui d'une section triangulaire, favorisant ainsi la dynamique du processus étudié, la géométrie carrée a été retenue dans cette étude (Fig. 2a) et d'un point de vue qualitatif, n'altère en rien la généralité des résultats obtenus.

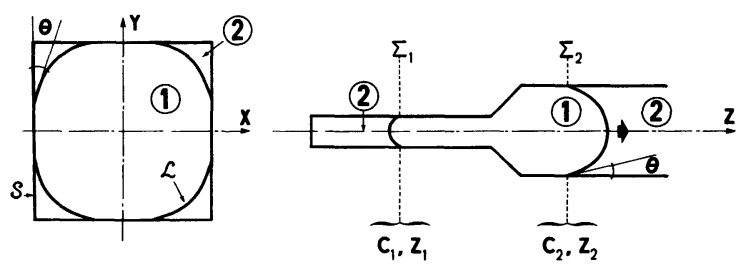

Fig. 2. - (a) Section transverse du capillaire ; (b) section longitudinale du capillaire.

[(a) Cross-section of the capillary tube ; (b) longitudinal section of the capillary tube.]

Cette géométrie permet ainsi l'écoulement simultané des deux fluides : le fluide mouillant occupe le domaine $\Omega_{2}$ constitué par les coins du capillaire modélisant pour un milieu poreux réel les anfractuosités des parois des pores, tandis que le fluide non mouillant occupe le domaine $\Omega_{1}$.

Le moteur de l'imbibition spontanée à contrecourant est essentiellement la capillarité. Pour modéliser ce processus, le capillaire utilisé présente une section longitudinale rétrécie (Fig. 2b) restituant ainsi l'effet des seuils et pores d'un milieu poreux.

Pour obtenir une telle géométrie, et pour en contrôler précisément les dimensions, un procédé original a été mis au point : une matrice en alliage (Dilver $\mathrm{P}_{1}$ ) est d'abord confectionnée, puis un verre de même coefficient de dilatation que l'alliage est moulé sur la matrice. Celui-ci est ensuite attaqué à l'eau régale (Legait, 1983). En suivant cette méthode, plusieurs capillaires de section carrée ont été fabriqués; le demi-côté d'une section allant de $0,5 \mathrm{~mm}$ à $2 \mathrm{~mm}$, la longueur totale du capillaire étant de l'ordre de $100 \mathrm{~mm}$. Une dimension inférieure à $0,5 \mathrm{~mm}$ pour le demi-côté d'une section n'est pas envisageable du fait d'une durée d'attaque à l'eau régale prohibitive, et d'un problème de déformation de la matrice.
1.2 CHOIX DES FLUIDES. - Quatre couples de fluides ont été utilisés. Le fluide mouillant est, pour toutes les expériences, de l'eau distillée $\left(\mu_{2}=\right.$ $\left.0,80 \mathrm{cP}, T=30^{\circ} \mathrm{C}\right)$. Les viscosités des fluides non mouillants et les tensions interfaciales des couples de fluides ainsi constitués sont répertoriés dans le tableau (Fig. 3).

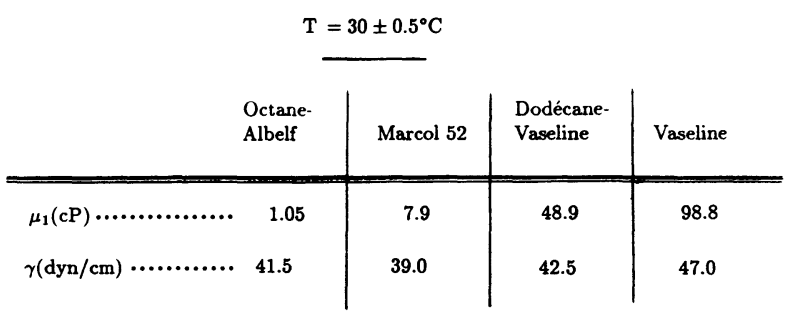

Fig. 3. - Caractéristiques physiques des fluides.

[Physical properties of fluids.]

1.3 Procédure EXPÉRIMENTALE. - Le capillaire placé horizontalement afin de s'affranchir de l'effet de la gravité et étant saturé de fluide mouillant, un globule de fluide non mouillant est produit par cisaillement dans un capillaire de section circulaire, coaxial avec le capillaire de section carrée (Fig. 4).

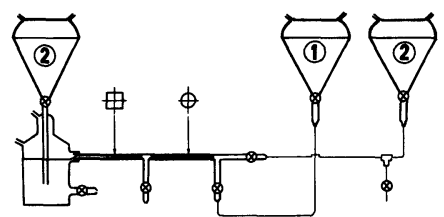

Fig. 4. - Montage expérimental : 1 fluide non mouillant ; 2 fluide mouillant.

[Experimental device: 1 non-wetting fluid; 2 wetting fluid.]

Le globule est ensuite positionné dans le capillaire de section carrée, de façon à ce que les deux ménisques $\Sigma_{1}$ et $\Sigma_{2}$ se trouvent de part et d'autre du rétrécissement (Fig. 2b). L'extrémité du capillaire de section carrée se trouvant du côté de la fine section est enfin obturée par une vanne, tandis que l'autre extrémité est mise au contact d'un plan d'eau affleurant de façon à alimenter le capillaire. Le globule se déplace alors spontanément sous l'action de la différence des pressions capillaires exercées sur les ménisques $\Sigma_{1}$ et $\Sigma_{2}$; le déplacement prend fin lorsque les deux ménisques retrouvent une même courbure. Au cours du mouvement spontané du globule, filmé et enregistré sur un magnétoscope, la position $Z_{1}$ du ménisque $\Sigma_{1}$ est repérée en fonction du temps. 


\section{Approche théorique.}

Nous adopterons dans toute la suite de cette étude, les hypothèses simplificatrices suivantes :

$\mathrm{H}_{1}$ : l'écoulement est axial sur toute section transverse du capillaire, i.e. le rétrécissement est suffisamment doux pour que les composantes transverses de la vitesse $\mathbf{u}_{i}$ de la phase $i(i=1,2)$, soient négligeables et que les pertes de charge au voisinage des ménisques soient faibles comparées au reste du globule;

$\mathrm{H}_{2}$ : l'écoulement est suffisamment lent pour que les termes d'inertie soient négligés, hypothèse qui a été vérifiée lors des simulations;

$\mathrm{H}_{3}$ : la courbure de l'interface fluide/fluide n'est fonction que de $z$, i.e. en toute section transverse du capillaire, la courbure garde une valeur constante.

Sous ces hypothèses, le système d'équations vérifiées par les pressions $\boldsymbol{P}_{\boldsymbol{i}}$ et la composante axiale $u_{i}$ de la vitesse $\mathbf{u}_{i}$, s'écrit dans chaque domaine $\Omega_{i}$ occupé par le fluide $i$ :

$$
\begin{aligned}
& i=1,2 ;\left(\partial_{x x}+\partial_{y y}\right) u_{i}=\frac{1}{\mu_{i}} \cdot \partial_{z} P_{i}, \\
& \forall(x, y) \in \Omega_{i}
\end{aligned}
$$

C.L.1 : $u_{1}=u_{2}=0$ le long de $S$,

surface du solide

$$
\begin{aligned}
\text { C.L. } 2: \mu_{1} \nabla u_{1} \cdot \mathbf{n}=\mu_{2} \nabla u_{2} \cdot \mathbf{n} ; & \\
& u_{1}=u_{2} \text { le long de } \mathcal{L}
\end{aligned}
$$

C.L.3 : $\left(P_{1}-P_{2}\right)(z)=\gamma C(z)$

où $\mathfrak{L}$ est l'interface fluide/fluide constituée de quatre arcs de cercle s'appuyant sur la surface du solide $S$ (Fig. 2a) - les calculs ayant montré que le flux dû à l'écoulement du fluide mouillant le long des parois du capillaire est négligeable devant celui le long des coins - $\mathbf{n}$ est le vecteur-unité normal (dirigé vers $\Omega_{1}$ ) à $\mathcal{L}$ en tout point de $\mathcal{L}, C(z)$ est la courbure de l'interface.

Compte tenu des hypothèses, la courbure $C_{i}$ des ménisques $\Sigma_{i}$ s'exprime en fonction de l'angle de mouillage $\theta$ à l'aide de la formule de Mayer (Mayer, 1965 ; Lenormand, 1981 ; Legait, 1983), vraie en statique et supposée telle d'après $\mathrm{H}_{2}$ :

$$
\left.\begin{array}{rl}
C_{i} & =\frac{F(\theta)}{R_{i}} \\
F(\theta) & =\frac{\theta+\cos ^{2}(\theta)-\pi / 4-\sin (\theta) \cos (\theta)}{\cos (\theta)-\sqrt{\pi / 4-\theta+\sin (\theta) \cos (\theta)}}
\end{array}\right\} .
$$

Du fait de $\mathrm{H}_{2}$, les gradients de pression $\partial_{z} P_{i}$ peuvent être considérés en première approximation comme constants sur une section transverse du capillaire (Legait, 1983). Ils s'expriment alors comme fonctions linéaires des débits $q_{i}$ des phases $i$, $i=1,2$ (annexe 1):

$$
(i=1,2 ; j=1,2) \frac{1}{\mu_{i}} \cdot \partial_{z} P_{i}=B_{i}^{j} \cdot \frac{q_{j}}{r^{4}(z)}
$$

où les coefficients $B_{i}^{i}$ ne dépendent que de l'angle de mouillage $\theta$, du contraste des viscosités $\bar{\mu}$ et de l'aire respective occupée par chacun des fluides dans une section transverse du capillaire.

L'intégration de (3) le long du globule conduit, compte tenu de la condition C.L.3, à :

$$
\begin{aligned}
\gamma\left[C_{2}-C_{1}\right]= & q_{1} \int_{z_{1}}^{z_{2}} \frac{B_{1}^{1} \mu_{1}-B_{2}^{1} \mu_{2}}{r^{4}(z)} \mathrm{d} z+ \\
& +q_{2} \int_{z_{1}}^{z_{2}} \frac{B_{1}^{2} \mu_{1}-B_{2}^{2} \mu_{2}}{r^{4}(z)} \mathrm{d} z .
\end{aligned}
$$

Le système formé par les équations (4) et l'équation (5) suivante (équation de conservation de la masse) :

$$
q_{1}+q_{2}=0
$$

permet de calculer les flux $q_{1}$ et $q_{2}$ pour une position donnée du globule, i.e. pour une valeur donnée de $Z_{1}$, connaissant la forme de la courbure $C(z)$ le long du globule (les coefficients $B_{i}^{j}$ dépendant de la répartition des fluides dans une section et donc de la forme de l'interface).

\section{Comparaison entre théorie et expérience.}

3.1 FORME DE L'INTERFACE FLUIDE/FLUIDE. - La cinétique du contre-courant peut être calculée moyennant une hypothèse sur la forme de l'interface. L'hypothèse la plus simple est de supposer que la courbure $C(z)$ est constante le long du globule hypothèse $\mathrm{H}_{4}$. L'équation (4) s'écrit alors :

$$
\begin{aligned}
\gamma\left[C_{2}-C_{1}\right]= & {\left[q_{1}\left(B_{1}^{1} \mu_{1}-B_{2}^{1} \mu_{2}\right)+\right.} \\
& \left.+q_{2}\left(B_{1}^{2} \mu_{1}-B_{2}^{2} \mu_{2}\right)\right] \int_{z_{1}}^{z_{2}} \frac{\mathrm{d} z}{r^{4}(z)} .
\end{aligned}
$$

Les équations (5) et (6) permettent alors de calculer le débit $Q$, valeur absolue commune des débits $q_{1}$ et $q_{2}$, pour un angle $\theta$ et une valeur de $\bar{\mu}$ donnés, en fonction de $Z_{1}$. Les résultats sont reportés dans un diagramme $\left(N_{\mathrm{c}}, \bar{Z}_{1}\right)$ (Fig. 5) où $N_{\mathrm{c}}$ est le nombre capillaire défini par :

et

$$
N_{\mathrm{c}}=\frac{\mu_{2} Q}{4 \gamma R_{1}^{2}}
$$

$$
\bar{Z}_{1}=\frac{Z_{1}}{R_{1}}
$$


Les courbes obtenues sous l'hypothèse $\mathrm{H}_{4}$, sont figurées par des tirets. La comparaison entre ces courbes et les points expérimentaux placés dans ce même diagramme montre que cette hypothèse est trop simpliste.

Nous supposons à présent que la courbure $C(z)$ est variable le long du globule - hypothèse $\mathrm{H}_{5}$ - sans expliciter davantage cette variation. Un calcul numérique montrant que la différence $B_{i}^{i}-B_{i}^{j}$ est proportionnelle à $C^{4}(z)$ (Legait, 1983), l'équation (3) écrite dans le cas du contre-courant entraîne la relation suivante entre les gradients de pression et les débits :

$$
\begin{aligned}
& i=1,2 ; \quad \partial_{z} P_{i}=a_{i}(z) q_{i} \\
& a_{i}(z)=\lambda_{i} C^{4}(z) ; \quad \lambda_{i}=\text { Cst } .
\end{aligned}
$$

La dérivation par rapport à $z$ de l'expression de la pression capillaire (condition C.L.3) associée à l'équation (7) écrite pour les deux phases, conduit à :

$$
\frac{\gamma}{\lambda} \cdot \frac{\mathrm{d} C}{C^{4}}=q_{1} \mathrm{~d} z
$$

où $\lambda=\lambda_{1}+\lambda_{2}$.

L'intégration par rapport à $z$ de l'équation (8) le long du globule, conduit à l'expression suivante de $q_{1}$ en fonction de $Z_{1}$ :

$$
q_{1}=\frac{\gamma\left(R_{1}^{3}-R_{2}^{3}\right)}{3 \lambda\left(Z_{2}-Z_{1}\right) F^{3}(\theta)}
$$

$Z_{2}$ étant déduit de la connaissance du volume du globule et de la valeur de $Z_{1}$, et $\lambda$ étant calculé par comparaison avec l'équation (3), pour les sections $S_{1}$ et $S_{2}$ du capillaire, sections pour lesquelles la valeur de la courbure est connue d'après la formule de Mayer (2). Il vient :

$$
\begin{gathered}
\lambda_{1}=\mu_{1} \cdot \frac{B_{1}^{1}-B_{1}^{2}}{F^{4}(\theta)} ; \quad \lambda_{2}=\mu_{2} \cdot \frac{B_{2}^{2}-B_{2}^{1}}{F^{4}(\theta)} ; \\
\lambda=\lambda_{1}+\lambda_{2} .
\end{gathered}
$$

L'intégration de l'équation (9) par rapport au temps fournit l'expression de $Z_{1}$ en fonction du temps :

$$
\begin{aligned}
& Z_{1}(t)=\beta(\theta)- \\
& -\sqrt{\beta^{2}(\theta)-\left[\alpha(\theta, \gamma, \bar{\mu})\left(R_{2}-R_{1}\right)^{2} / 2 s_{1}\right]} t
\end{aligned}
$$

avec $t \leqslant t_{\max }, t_{\max }$ étant atteint lorsque $Z_{1}=l$, et où :

- $\beta$ dépend des caractéristiques géométriques du capillaire, de l'angle de mouillage $\theta$ par l'intermédiaire de $F(\theta)$ et du volume du globule;

- $\alpha$ dépend des caractéristiques géométriques du capillaire, de l'angle de mouillage $\theta$ par l'intermédiaire de $\lambda$ et $F(\theta)$, de la tension interfaciale $\gamma$ et du contraste des viscosités $\bar{\mu}$ par l'intermédiaire de $\lambda$;

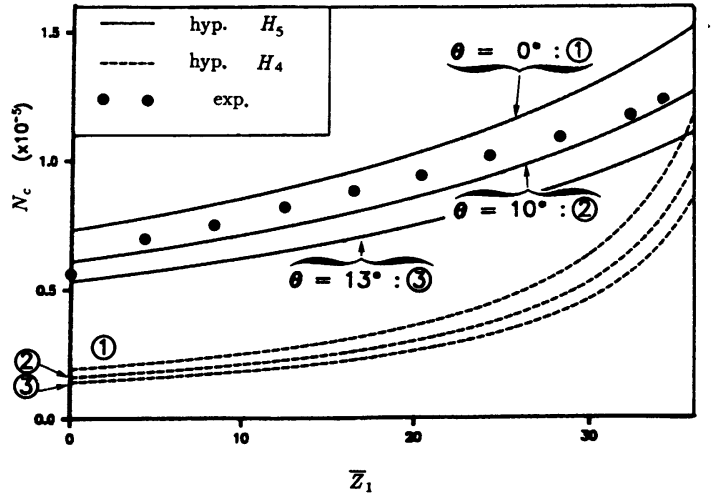

Fig. 5. - Comparaison des hypothèses $\mathbf{H}_{4}$ et $\mathrm{H}_{5}$ à l'expérience, influence de l'angle de mouillage.

[Comparison of assumptions $\mathrm{H}_{4}$ and $\mathrm{H}_{5}$ with experimental results, influence of contact angle.]

(les expressions de $\beta(\theta)$ et $\alpha(\theta, \gamma, \bar{\mu})$ sont données dans l'annexe 2);

- $s_{1}$ est la saturation en fluide non mouillant dans une section transverse.

En portant les points expérimentaux sur un diagramme $\left(Z_{1}, t\right)$ et en les comparant avec les valeurs de $Z_{1}$ fonction du temps obtenues par l'équation (10) pour différentes valeurs de $\theta$, une valeur de l'angle de mouillage peut être déterminée (Fig. 6). Pour une valeur de $\theta$ voisine de $15^{\circ}$, il y a pour toutes les expériences une bonne corrélation entre théorie et expérience. Cette valeur de l'angle de mouillage, qui apparaît comme une valeur de calage entre le modèle et l'expérience est cependant tout à fait comparable aux mesures expérimentales, le plus souvent indirectes, d'angle de mouillage apparent dans un processus d'imbibition (Ngan et al., 1982).

Les courbes $\left(q_{1}, Z_{1}\right)$ obtenues par l'équation (9) et reportées sur le diagramme (Fig. 5) (traits continus) concordent cette fois avec les points expérimentaux et montrent que l'hypothèse $\mathrm{H}_{5}$ est satisfaisante.

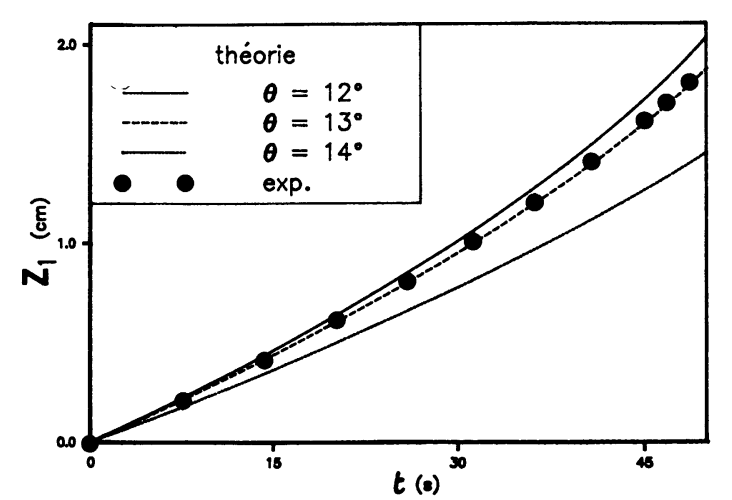

Fig. 6. - Cinétique du contre-courant, comparaison théorie/expérience, influence de l'angle de mouillage.

[Kinetics of counter-current flow, theory versus experiment, influence of contact-angle.] 


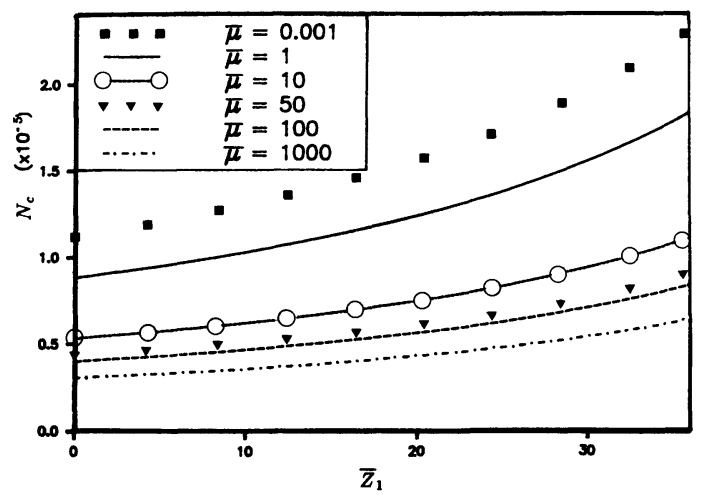

Fig. 7. - Influence du contraste des viscosités sur la dynamique du contre-courant.

[Influence of viscosity ratio on dynamics of counter-current flow.

3.2 INFLUENCE DE L'ANGLE DE MOUIllaGe. - La modélisation de l'écoulement à contre-courant dans un capillaire de section carrée, dont la géométrie est connue, étant satisfaisante pour plusieurs cas de figures, l'influence des différents paramètres du système peut être examinée au moyen du modèle (Kalaydjian et al., 1987a) : un contraste de viscosité élevé abaisse la dynamique du contre-courant (Fig. 7), cette dynamique étant en revanche favorisée par une forte tension interfaciale et une taille de globule importante (Eq. (9)).

En ce qui concerne l'influence de la mouillabilité, la figure 6 montre que la dynamique du contrecourant est favorisée par un angle $\theta$ faible, puisque pour toute position du globule, le nombre capillaire $N_{\mathrm{c}}$ est d'autant plus élevé que l'angle de mouillage est faible. D'autre part, la dynamique du contrecourant est nulle pour un angle de mouillage de $\pi / 4$ puisque pour cet angle, l'espace des coins permettant l'écoulement du fluide mouillant s'annule.

\section{Couplage et perméabilités relatives.}

4.1 QuANTIFICATION DU COUPlage. - L'écriture classique de la loi de Darcy polyphasique ne prend pas explicitement en compte les couplages entre phases : le gradient de pression d'une phase contrôle nécessairement l'écoulement de l'autre. La prise en compte de ce couplage entraîne alors une nouvelle formulation des lois d'écoulement faisant apparaître des termes croisés traduisant le couplage :

$$
\left.\begin{array}{l}
u_{1}=\frac{k \cdot k_{r 11}}{\mu_{1}}\left(-\partial_{z} P_{1}\right)+\frac{k \cdot k_{r 12}}{\mu_{2}}\left(-\partial_{z} P_{2}\right) \\
u_{2}=\frac{k \cdot k_{r 21}}{\mu_{1}}\left(-\partial_{z} P_{1}\right)+\frac{k \cdot k_{r 22}}{\mu_{2}}\left(-\partial_{z} P_{2}\right)
\end{array}\right\} .
$$

Ecrite dans le cas monodimensionnel, cette équation se généralise sans peine au cas tridimensionnel en introduisant des perméabilités d'ordre tensoriel. Seuls trois des quatre coefficients de la matrice des perméabilités $\left(k_{r i j}\right)$ sont à calculer puisque d'après les relations de réciprocité d'Onsager :

$$
k_{r 21}=\bar{\mu} k_{r 12} \text {. }
$$

Cette représentation des lois d'écoulement a été suggérée de façon théorique à de nombreuses reprises (Auriault et al., 1987 ; de Gennes, 1983 ; Marle, 1982 ; Whitaker, 1986), cependant, les termes $k_{r i j}(i \neq j)$ n'ont à notre connaissance, jamais encore été déterminés quantitativement.

L'équation (3) permet de calculer explicitement les termes $k_{r i j}$ en les reliant aux coefficients $B_{i}^{i}$. Il vient :

$$
\begin{aligned}
& k_{r i i}=\frac{-B^{*} B_{j}^{j}}{\operatorname{det}\left(B_{i}^{j}\right)} \\
& k_{r i j}=\frac{B^{*} B_{i}^{j}}{\operatorname{det}\left(B_{i}^{i}\right)} \quad(i, j=1,2 ; i \neq j) .
\end{aligned}
$$

Les variations des perméabilités relatives $k_{r i j}$ peuvent donc être calculées en fonction de la saturation (Fig. 8a). Les calculs sont effectués pour un angle de mouillage nul et un contraste de viscosité donné $\bar{\mu}$ égal à l'unité. Pour des saturations inférieures à 0,78 , valeur de la saturation pour laquelle l'interface fluide-fluide n'est plus en contact avec la paroi solide, les variations des perméabilités relatives sont obtenues en considérant que la phase fluide non mouillante dans une section droite du capillaire, occupe un disque centré sur l'axe $z$.

Cette figure permet de constater que les termes de couplage (égaux pour $\bar{\mu}=1$ ) ne sont pas négligeables devant les deux termes diagonaux simultanément : pour les valeurs extrêmes de la saturation, les termes de couplage peuvent être de l'ordre de grandeur de l'un des termes croisés, et pour des valeurs moyennes de la saturation, si le couplage est bien inférieur aux termes diagonaux, il n'est pas négligeable.

L'étude de l'influence du contraste des viscosités, dans cette formulation (Kalaydjian et al., 1987b) montre un effet de lubrification (le terme $k_{r 11}$ croissant avec $\bar{\mu}$ ), résultat obtenu sur d'autres types de capillaires (Singhal et al., 1970) et sur un milieu poreux (Danis et al., 1983).

\subsection{Application au CAS du CONTRE-Courant. -} La généralisation des résultats obtenus sur le type de capillaire utilisé est tentante, dans la mesure où les valeurs mesurées du nombre capillaire $N_{\mathrm{c}}$ sont équivalentes à celles qui ont cours en milieu poreux. Une comparaison entre les écoulements en capillaire et en milieu poreux peut être effectuée en utilisant les relations entre gradients de pression et flux établies pour le capillaire qui permettent de calculer 

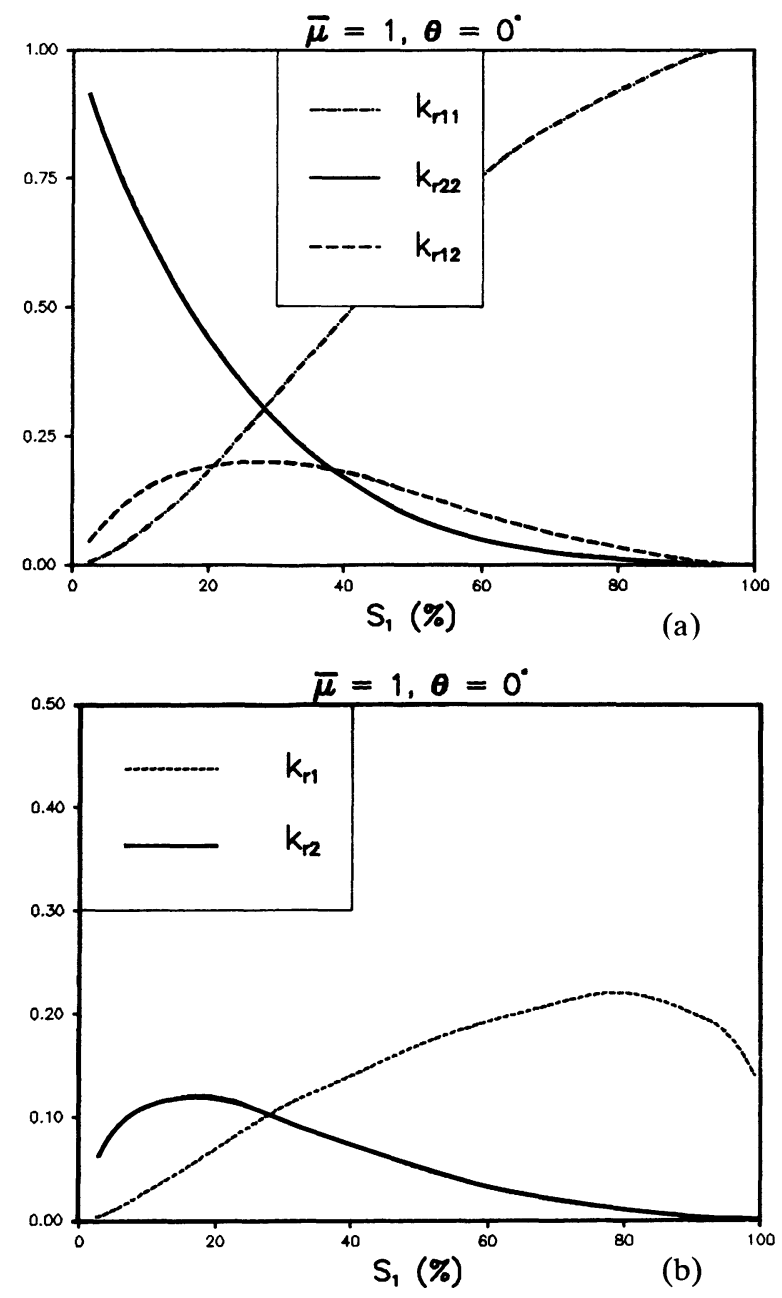

Fig. 8. - (a) Matrice des perméabilités relatives ; (b) perméabilités relatives à contre-courant en capillaire.

[(a) Relative permeability matrix ; (b) relative permeability curves for counter-current flow in the capillary tube.]

par analogie avec un milieu poreux, des perméabilités relatives. Dans le cas du contre-courant, une équivalence entre les deux descriptions, classique et avec couplage, peut être obtenue (Annexe 3).

Le gradient de pression et le débit exprimés pour une phase donnée vérifient l'équation suivante obtenue en portant l'équation (5) dans l'équation (3) :

$$
\partial_{z} P_{i}=\varepsilon_{i} \mu_{i} \frac{B_{i}^{1}-B_{i}^{2}}{r^{4}(z)} q_{i} \quad i=1,2
$$

où $\varepsilon_{i}=\operatorname{sgn}(3 / 2-i)$.

L'expression de $q_{i}(i=1,2)$, en fonction d'une vitesse $u_{i}$ moyennée sur la totalité de la section droite :

$$
q_{i}=4 r^{2}(z) u_{i}
$$

permet d'écrire l'équation (12) sous la forme suivante :

$$
u_{i}=\frac{1}{\mu_{i}} \cdot \frac{r^{2}(z)}{4 \varepsilon_{i}\left(B_{i}^{1}-B_{i}^{2}\right)} \partial_{z} P_{i} \quad i=1,2 .
$$

L'analogie avec la loi de Darcy polyphasique, conduit à l'expression des perméabilités relatives sous la forme :

$$
k \cdot k_{r i}=-\frac{r^{2}(z)}{4 \varepsilon_{i}\left(B_{i}^{1}-B_{i}^{2}\right)} \quad i=1,2 .
$$

La perméabilité $k$ étant calculée par $k=-r^{2}(z) / 4 B^{*}$, où $B^{*}$ est obtenu à partir du profil des vitesses dans le cas monophasique, il vient :

$$
k_{r i}=\varepsilon_{i} \frac{B^{*}}{B_{i}^{1}-B_{i}^{2}} \quad i=1,2 .
$$

Les courbes obtenues (Fig. 8b) représentent les variations des perméabilités relatives $k_{r i}$ en fonction de la saturation $s_{1}$, pour un angle $\theta$ nul et pour un contraste de viscosités $\bar{\mu}$ égal à 1 . Ces courbes présentent une forme non monotone qui est inhabituelle. Ceci provient d'une part du fait que pour des saturations extrêmes en huile le contre-courant ne peut se produire, et d'autre part d'une forte interaction entre les fluides dans le type de micromodèle utilisé, qui se traduit nécessairement en terme de couplage.

L'influence du contraste des viscosités sur le type d'écoulement peut être retrouvée en examinant la variation des courbes de perméabilité relative pour des valeurs croissantes de $\bar{\mu}$ (Kalaydjian et al., 1987b) : la courbe $k_{r 1}$ croît tandis que la courbe $k_{r 2}$ décroît. Cette variation de $k_{r 2}$ correspond bien à un déplacement ralenti, à $\bar{\mu}$ croissant, d'un globule de fluide non mouillant en imbibition spontanée à contre-courant, résultat déjà signalé dans la section 3 de cette étude.

4.3 COMPARAISONS ENTRE MILIEU POREUX ET MICROMODÈLE. - Les limites de l'utilisation en milieu poreux des résultats obtenus sur micromodèle doivent être-soulignées : des conditions de calcul restrictives dues à l'approximation de Stokes ne peuvent traduire l'existence d'écoulements rapides de globules, la surface de l'interface liquide/liquide est, proportionnellement à la surface spécifique du solide, plus grande dans le cas du type de capillaire utilisé que dans celui d'un milieu poreux, enfin, l'écoulement simultané des deux fluides dans un même pore n'est pas le seul type d'écoulement possible en imbibition spontanée, le déplacement de type piston en milieu poreux étant bien connu. De ce fait, l'extrapolation au milieu poreux des résultats concernant les variations des perméabilités relatives en fonction de la saturation (résultats obtenus dans cette étude) ne peut être menée qu'avec précaution. Cependant, pour un milieu suffisamment homogène, la mise en mouvement d'un globule de fluide non mouillant par progression du fluide mouillant le long des parois des pores, tel que le simule le micromodèle, doit jouer un rôle non négligeable. 
Les mesures comparées des perméabilités relatives à co-courant et à contre-courant sur un même milieu poreux et avec un même couple de fluides ont rarement été menées. Une telle étude expérimentale (Lelièvre, 1967) a cependant montré que les perméabilités relatives classiques mesurées en régime permanent à co-courant et à contre-courant différaient sensiblement, les perméabilités mesurées à contrecourant étant sensiblement inférieures à celles à cocourant (de l'ordre de $25 \%$ ). Une approche telle que celle qui a été développée dans le cadre des écoulements en capillaire, peut expliquer cette différence par un couplage même faible. Ceci tend à montrer que même dans le cas d'un milieu poreux, l'origine différente des processus physiques mis en jeu dans les deux types de déplacement diphasique (couplage entre phases fluides négligeables à cocourant mais prépondérant à contre-courant) peut expliquer une différence sensible entre les perméabilités à co-courant et à contre-courant.

\section{Conclusions.}

L'étude du déplacement à contre-courant en imbibition spontanée d'un globule de fluide non mouillant dans un capillaire de sections carrées, rendu possible par la géométrie spéciale du capillaire, a permis d'exhiber un fort couplage entre les phases fluides, prépondérant dans ce type de déplacement. L'écriture des équations du système, comparées avec les lois classiques de Darcy qui régissent les écoulements en milieu poreux, a conduit à définir une matrice de perméabilités prenant explicitement en compte ce couplage. L'application de cette nouvelle formulation dans le cas d'un milieu poreux a permis d'expliquer les différences observées entre les perméabilités mesurées à co-courant et à contre-courant et montre finalement la généralité des résultats obtenus sur capillaire.

\section{Annexe 1.}

RÉSOLUTION DU SYSTÈME (1). — La modélisation d'un écoulement diphasique dans un capillaire de section carrée conduit au système suivant :

$i=1,2 ;\left(\partial_{x x}+\partial_{y y}\right) u_{i}=\frac{1}{\mu_{i}} \cdot \partial_{z} P_{i}, \quad \forall(x, y) \in \Omega_{i}$

C.L.1 : $u_{1}=u_{2}=0$ le long de $S$

C.L. $2: \mu_{1} \nabla u_{1} \cdot \mathbf{n}=\mu_{2} \nabla u_{2} \cdot \mathbf{n} ; u_{1}=u_{2}$ le long de $\mathcal{L}$

où $1 / \mu_{i} \cdot \partial_{z} P_{i}$ est une constante. Une base de solutions de ce système est obtenue en résolvant les deux sous-problèmes $\left(I_{j}\right), j=1,2$ suivants :

$$
\left(I_{j}\right)\left\{\begin{array}{c}
i=1,2 ;\left(\partial_{\bar{x} \bar{x}}+\partial_{\overline{y y}}\right) v_{i j}=\delta_{i j} \\
\text { C.L.1 }: v_{1 j}=v_{2 j}=0 \text { le long de } S \\
\text { C.L.2: } \mu_{1} \nabla v_{1 j} \cdot n=\mu_{2} \nabla v_{2 j} \cdot n ; \\
v_{1 j}=v_{2 j} \text { le long de } \mathcal{L}
\end{array}\left(\mathrm{A}_{1} .2\right)\right.
$$

où $\bar{x}=x / r(z) ; \bar{y}=y / r(z), r(z)$ étant le demi-côté de la section du capillaire à la cote $z$, et $\delta_{i j}$ est le symbole de Kronecker.

Les solutions $v_{i j}$ des systèmes $\left(I_{j}\right)$ formant par construction une base de solutions du système (1), la solution générale de ce système s'écrit alors :

$$
u_{i}(x, y, z)=\sum_{j=1,2} A_{j}(z) v_{i j}(x, y)
$$

la comparaison de $\left(\mathrm{A}_{1} .1\right),\left(\mathrm{A}_{1} .2\right)$ et $\left(\mathrm{A}_{1} .3\right)$ permettant de calculer $A_{j}(z)$ :

$$
A_{j}(z)=\frac{r^{2}(z)}{\mu_{j}} \cdot \partial_{z} P_{j}
$$

d'où :

$$
u_{i}(x, y, z)=\sum_{j=1,2} v_{i j}(x, y) \frac{r^{2}(z)}{\mu_{j}} \partial_{z} P_{j}
$$

Les débits volumes $q_{i}$ étant définis par :

$$
q_{i}=\int_{\Omega_{i}} u_{i} \mathrm{~d} x \mathrm{~d} y
$$

il vient :

$$
q_{i}=r^{4}(z) \sum_{j=1,2} C_{i j} 1 / \mu_{j} \partial_{z} P_{j}
$$

où :

$$
C_{i j}=\int_{\Omega_{i}} v_{i j} \mathrm{~d} \bar{x} \mathrm{~d} \bar{y} .
$$

L'équation (3) se déduit alors de $\left(A_{1} .7\right)$ en appelant $\left(B_{i}^{i}\right)$ la matrice inyerse de $\left(C_{i}^{j}\right)$.

La résolution des deux systèmes $\left(I_{j}, j=1,2\right)$ permet de calculer les vitesses $v_{i j}$ en tout point d'une section transverse renormalisée (par raison de symétrie, il suffit de résoudre le problème sur $1 / 8 \mathrm{de}$ section). Ces calculs ont été effectués par une méthode aux éléments finis, à l'aide des logiciels MOSAIC et MEF. La résolution nécessite un maillage d'autant plus fin que $\bar{\mu}$ est fort du fait de la condition en gradient imposée sur l'interface $\mathcal{L}$.

\section{Annexe 2.}

CAlCUl DES FACTEURS DE FORME $\beta(\theta)$ ET $\alpha(\bar{\mu}, \gamma, \theta)$. - Pour obtenir l'équation (10) à partir de l'équation (9), il faut exprimer $Z_{2}$ en fonction de $Z_{1}$. Ce calcul permet alors d'expliciter les expressions de $\alpha$ et $\beta$.

Le calcul de $Z_{2}(t)$ en fonction de $Z_{1}(t)$ est effectué en écrivant la conservation du volume du globule d'huile au cours du mouvement. 
Soit $s_{1}$, la saturation en fluide non mouillant dans une section transverse du capillaire. Lorsque le globule d'huile se trouve en amont du rétrécissement, i.e. dans la fine section du capillaire, son volume est :

$$
\vartheta=4 R_{1}^{2} L s_{1}+2 v_{1}
$$

où $L$ est la longueur du globule et $v_{i}$ le volume de la calotte sphérique du ménisque $\Sigma_{i}$ qui constitue l'extrémité du globule.

Lors du déplacement du globule, les ménisques se trouvent de part et d'autre du rétrécissement et $v$ se calcule de la façon suivante :

$$
\begin{aligned}
\vartheta=v_{1} & +v_{2}+\left(l-Z_{1}\right) 4 R_{1}^{2} s_{1}+ \\
& +\left[Z_{2}-(l+d)\right] 4 R_{2}^{2} s_{1}+\int_{l}^{l+d} 4 s_{1} r^{2}(z) \mathrm{d} z
\end{aligned}
$$

l'intégrale, représentant le volume du globule compris dans le rétrécissement, ayant l'expression suivante en fonction des caractéristiques géométriques du capillaire :

$$
\int_{l}^{l+d} 4 s_{1} r^{2}(z) \mathrm{d} z=4 s_{1} \frac{d}{R_{2}-R_{1}} \cdot \frac{R_{2}^{3}-R_{1}^{3}}{3} .
$$

En égalant les deux expressions obtenues pour le volume $v$, il vient :

$$
\begin{aligned}
& Z_{2}=\left(Z_{1}+L\right)\left(\frac{R_{1}}{R_{2}}\right)^{2}+\frac{1}{R_{2}^{2}}\left[l\left(R_{2}^{2}-R_{1}^{2}\right)+\right. \\
& \left.+d\left(R_{2}^{2}-\frac{d}{R_{2}-R_{1}} \cdot \frac{R_{2}^{3}-R_{1}^{3}}{3}\right)\right]+\frac{1}{R_{2}^{2}}\left(\frac{v_{1}-v_{2}}{4 s_{1}}\right) .
\end{aligned}
$$

Le calcul du dernier terme, correspondant à la variation du volume du ménisque aval, est simplifié en considérant que la surface délimitant ce volume est une portion de calotte sphérique s'appuyant sur un cercle inscrit dans la section carrée. Un calcul plus exact, mais plus compliqué qui tiendrait compte de la forme exacte de la trace du ménisque sur la section $S_{2}$, serait de scinder la calotte en deux régions, l'une à bord circulaire, l'autre à bord rectiligne, et d'intégrer ensuite dans ces deux domaines.

Sous cette hypothèse, l'erreur de calcul faite est faible et est d'autant plus négligeable que la portion du globule entre les deux sections $S_{1}$ et $S_{2}$ est longue devant la taille des ménisques, ce qui est nécessaire pour pouvoir faire une expérience de longue durée et avoir ainsi plusieurs points expérimentaux.

L'expression du dernier terme s'écrit alors :

$$
\begin{aligned}
\frac{1}{R_{2}^{2}}( & \left.\frac{v_{1}-v_{2}}{4 s_{1}}\right)=\frac{1}{4 R_{2}^{2} s_{1}} \cdot \frac{\pi\left(R_{1}^{3}-R_{2}^{3}\right)}{F^{3}(\theta)} \times \\
& \times\left[\frac{2}{3}-F(\theta) \operatorname{tg}(\theta)\left(1-\frac{1}{3} F^{2}(\theta) \operatorname{tg}^{2}(\theta)\right)\right] .
\end{aligned}
$$

Par conséquent l'expression de $Z_{2}$ en fonction de $Z_{1}$ devient :

$$
Z_{2}=\left(Z_{1}+L\right)\left(\frac{R_{1}}{R_{2}}\right)^{2}+\frac{f(\theta)}{R_{2}^{2}}
$$

où :

$$
\begin{aligned}
f(\theta) & =\frac{1}{4 s_{1}} \cdot \frac{\pi\left(R_{1}^{3}-R_{2}^{3}\right)}{F^{3}(\theta)} \times \\
& \times\left[\frac{2}{3}-F(\theta) \operatorname{tg}(\theta)\left(1-\frac{1}{3} F^{2}(\theta) \operatorname{tg}^{2}(\theta)\right)\right] \\
& +\left(R_{2}^{2}-R_{1}^{2}\right) l+\left(R_{2}^{2}-\frac{d}{R_{2}-R_{1}} \cdot \frac{R_{2}^{3}-R_{1}^{3}}{3}\right) d .
\end{aligned}
$$

En donnant à $\alpha$ et $\beta$ respectivement les valeurs suivantes :

$$
\begin{aligned}
& \alpha=\frac{\gamma}{3 \lambda F^{3}(\theta)} \cdot \frac{\left(R_{2}^{3}-R_{1}^{3}\right)}{\left(R_{2}^{2}-R_{1}^{2}\right)} \\
& \beta=\frac{f(\theta)+L R_{1}^{2}}{R_{2}^{2}-R_{1}^{2}}
\end{aligned}
$$

ceci permet d'écrire l'équation (9) sous la forme :

$$
q_{1}=\frac{\alpha R_{2}^{2}}{Z_{1}-\beta}
$$

ce qui conduit à l'équation (10).

\section{Annexe 3.}

EQUIVALENCE ENTRE LA DESCRIPTION CLASSIQUE ET LA DESCRIPTION AVEC COUPLAGE DANS LE CAS DU CONTRE-COURANT. - L'équivalence entre les deux descriptions est obtenue ainsi : partant des systèmes d'équations décrivant les écoulements dans le cas classique et dans le cas avec couplage, une équation aux dérivées partielles vérifiée par la saturation est obtenue pour chacun de ces deux cas ; il y a équivalence entre les deux approches si et seulement si les coefficients des deux E.D.P. sont égaux.

a) Système d'équations dans le cas classique. Dans le cas classique monodimensionnel, le système différentiel décrivant l'écoulement est le suivant :

$$
\begin{array}{lll}
u_{1} & =-k_{1}\left(\partial_{z} P_{1}-\rho_{1} g\right) & \left(\mathrm{A}_{3} \cdot 1\right) \\
u_{2} & =-k_{2}\left(\partial_{z} P_{2}-\rho_{2} g\right) & \left(\mathrm{A}_{3} .2\right) \\
u_{1}+u_{2} & =0 & \left(\mathrm{~A}_{3} .3\right) \\
s_{1}+s_{2} & =1 & \left(\mathrm{~A}_{3} \cdot 4\right) \\
\partial_{z} u_{i}+\phi \partial_{t} s_{i} & =0 & \left(\mathrm{~A}_{3} .5\right) \\
P_{1}-P_{2} & =P_{c}\left(s_{1}\right) & \left(\mathrm{A}_{3} \cdot 6\right)
\end{array}
$$

où les coefficients $k_{i}$ sont les mobilités qui sont 
définies à partir des perméabilités de la façon suivante :

$$
k_{i}=\frac{k \cdot k_{r i}}{\mu_{i}} \quad i=1,2 .
$$

En combinant les équations $\left(A_{3} .1\right),\left(A_{3} .2\right)$ et $\left(A_{3} .3\right)$, il vient :

$$
k_{1}\left(\partial_{z} P_{1}-\rho_{1} g\right)+k_{2}\left(\partial_{z} P_{2}-\rho_{2} g\right)=0
$$

puis, en utilisant $\left(\mathrm{A}_{3} .6\right)$ :

$\partial_{z} P_{1}-\rho_{1} g=\frac{k_{2}}{k_{1}+k_{2}}\left(\left(\rho_{\mathrm{w}}-\rho_{0}\right) g+\partial_{z} P_{\mathrm{c}}\right) .\left(\mathrm{A}_{3} .7\right)$

D'où $s_{\mathrm{w}}$ vérifie l'E.D.P. suivante :

$\partial_{z}\left[\frac{k_{1} k_{2}}{k_{1}+k_{2}}\left(\partial_{z} P_{\mathrm{c}}+\left(\rho_{\mathrm{w}}-\rho_{\mathrm{o}}\right) g\right)\right]+\phi \partial_{t} s_{2}=0$.

b) Système d'équations dans le cas avec couplage. Le système d'équations ne diffère du précédent que par la formulation des lois de Darcy (Eq. (11)), les équations $\left(\mathrm{A}_{3} .1\right)$ et $\left(\mathrm{A}_{3} .2\right)$ devenant :

$$
\left\{\begin{array}{l}
u_{1}=k_{11}\left(-\partial_{z} P_{1}\right)+k_{12}\left(-\partial_{z} P_{2}\right) \\
u_{2}=k_{12}\left(-\partial_{z} P_{1}\right)+k_{22}\left(-\partial_{z} P_{2}\right)
\end{array}\right.
$$

où les coefficients $k_{i j}$ sont définis à partir des coefficients $k_{r i j}$ de la façon suivante :

$$
k_{i j}=\frac{k \cdot k_{r i j}}{\mu_{j}} \quad i, j=1,2
$$

et $k_{12}=k_{21}$ d'après les relations de réciprocité d'Onsager.

En posant,

$$
\left\{\begin{array}{l}
\Sigma=k_{11}+k_{22}+2 k_{12} \\
\Delta=k_{11} k_{22}-k_{12}^{2}
\end{array}\right.
$$

il vient :

$\partial_{z} P_{1}-\rho_{1} g=\frac{k_{12}+k_{22}}{\Sigma}\left(\left(\rho_{\mathrm{w}}-\rho_{\mathrm{o}}\right) g+\partial_{z} P_{\mathrm{c}}\right)$

puis,

$\partial\left[\frac{\Delta}{\Sigma}\left(\left(\rho_{\mathrm{w}}-\rho_{\mathrm{o}}\right) g+\partial_{z} P_{\mathrm{c}}\right)\right]+\phi \partial_{t} s_{\mathrm{w}}=0 .\left(\mathrm{A}_{3} \cdot 10\right)$

L'équivalence entre les deux descriptions est vérifiée si les E.D.P. $\left(A_{3} .7\right)$ et $\left(A_{3} .9\right)$ d'une part et $\left(A_{3} .8\right)$ et $\left(A_{3} .10\right)$ d'autre part ont mêmes coefficients. Ceci entraîne :

$$
k_{i}=\frac{\Delta}{k_{j j}+k_{i j}} \quad i, j=1,2 .
$$

\section{Bibliographie}

[1] Auriault, J. L. et al., Remarques sur la loi de Darcy pour les écoulements diphasiques en milieu poreux, J. Theor. Appl. Mech., n spécial (1986) 141-156.

[2] DARCy, H., Les fontaines publiques de la ville de Dijon (Ed. Dalmont, Paris) 1856.

[3] DE GENNES, P.-G., Theory of slow biphasic flows in porous media, Phys. Chem. Hydr. 4, 2 (1983) 175-185.

[4] (a) KAlAydjian, F. et al., Ecoulement lent à contrecourant de deux fluides non miscibles dans un capillaire présentant un rétrécissement, C.R. Hebd. Séan. Acad. Sci. Paris 304 (1987) 869872 ;

(b) Kalaydjian, F. et al., Perméabilités relatives couplées dans des écoulements en capillaire et en milieu poreux, C.R. Hebd. Séan. Acad. Sci. Paris 304 (1987) 1035-1038.

[5] LEGAIT, B., Interprétation de certains types d'écoulements diphasiques en milieu poreux à partir des écoulements en capillaires, thèse présentée à l'université de Bordeaux I (1983).

[6] LelièVre, R. F., Etude d'écoulements diphasiques permanents à contre-courants en milieu poreux, comparaison avec les écoulements de même sens, thèse présentée à l'université de Toulouse (1966).

[7] LeNORMAND, R., Déplacements polyphasiques en milieu poreux sous l'influence des forces capillaires. Etude expérimentale et modélisation de type percolation, thèse présentée à l'université de Toulouse (1981).

[8] Lenormand, R., Role of roughness and edges during imbibition in square capillaries, Soc. Pet. Eng. $\mathrm{n}^{\circ} 13264$ (1984).

[9] Marle, C. M., Ecoulements monophasiques en milieu poreux, Rev. Inst. Fr. Pet. 22 (1967) 14711509.

[10] MARLE, C. M., On macroscopic equations governing multiphase flow with diffusion and chemical reactions in porous media, Int. J. Eng. Sci. 20 (1982) 643-662.

[11] MAYeR, R. P. et al., Mercury porosimetry. Breakthrough pressure for penetration between packed spheres, J. Colloid Int. Sci. 20 (1965) 893-911.

[12] NGAN, C. G. et al., On the nature of the dynamic contact angle : an experimental study, J. Fluid Mech. 118 (1982) 27-40. 
[13] Wyckoff, R. D. et al., Physics 7 (1936) 325.

[14] Rose, W., Second thoughts on Darcy's law, Iran. Pet. Inst. Bull. 48 (1971) 25-30.
[15] Singhal, A. K. et al., Two-phase flow through a non-circular capillary at low Reynolds numbers, J. Can. Pet. (July-Sept. 1970) 197-205. 